\title{
New single resistance controlled oscillators employing a reduced number of unity-gain cells
}

\author{
S. S. Gupta and R. Senani ${ }^{a)}$ \\ Analog Signal Processing Research Lab., Division of Electronics and Communi- \\ cation Engineering, Netaji Subhas Institute of Technology, Sector-3, Dwarka, New \\ Delhi 110 075, India
}

a)senani@nsit.ac.in

\begin{abstract}
Recently, the authors introduced two new second-order single-resistance-controlled oscillators (SRCO) employing four/three unity-gain cells (unity-gain voltage followers and unity-gain current followers). In this communication, a novel family of SRCOs has been presented which employ a reduced number of (only two) unity-gain cells as active elements. The workability of the new circuits has been confirmed by the SPICE simulations of their CMOS-implementable versions.
\end{abstract}

Keywords: Sinusoidal Oscillators, Voltage Followers, Current Followers

Classification: Analog circuit design

\section{References}

[1] R. Senani and S. S. Gupta, "Novel sinusoidal oscillators using only unitygain voltage followers and current followers," IEICE Electron. Express, vol. 1, no. 13, pp. 404-409, Oct. 10, 2004.

[2] R. Senani, "New RC-active oscillator configuration employing unity-gain amplifiers," Electron. Lett., vol. 21, no. 20, pp. 889-891, Sept. 1985.

[3] N. Boutin, "Synthesis of oscillator circuits employing only one unity-gain amplifier," Electron. Lett., vol. 22, no. 1, pp. 22-23, Jan. 1986.

[4] M. T. Abuelma'atti, "Grounded-capacitor current-mode oscillator using single current follower," IEEE Trans. Circuits Syst. I, vol. 39, no. 12, pp. 1018-1020, Dec. 1992.

[5] M. T. Abuelma'atti, "New current-mode oscillators using a single unitygain current follower," Active Passive Elec. Comp., vol. 18, pp. 151-157, 1995.

[6] M. T. Abuelma'atti, "New current-mode oscillators using unity-gain current followers," Active Passive Elec. Comp., vol. 18, pp. 159-164, 1995.

[7] M. T. Abuelma'atti and H. Al-Daghreer, "New single-resistor-controlled sinusoidal oscillator circuit using unity-gain current followers," Active Passive Elec. Comp., vol. 20, pp. 105-109, 1997.

[8] M. T. Abuelma'atti and M. H. Khan, "Ten faster op-amp oscillators," Electron. Wireless W., vol. 93, no. 1620, pp. 1053-1054, 1987. 
[9] J. J. Chen, C. C. Chen, H. W. Tsao, and S. I. Liu, "Current-mode oscillators using single current follower," Electron. Lett., vol. 27, no. 22, pp. 2056-2059, Oct. 1991.

[10] R. Senani and S. S. Gupta, "Synthesis of single-resistance-controlled oscillators using CFOAs: simple state-variable approach," IEE Proc. Circ. Dev. Syst., vol. 144, no. 2, pp. 104-106, April 1997.

[11] S. Celma, J. Sabadell, and P. A. Martinez, "Universal filter using unitygain cells," Electron. Lett., vol. 31, no. 21, pp. 1817-1818, Oct. 1995.

[12] M. T. Abuelma'atti and M. A. Al-Qahtani, "Current-mode universal filters using unity-gain cells," Electron. Lett., vol. 32, no. 12, pp. 10771079, June 1996.

[13] H. A. Alzaher and M. Ismail, "Current-mode universal filter using unitygain cells," Electron. Lett., vol. 35, no. 25, pp. 2198-2200, Dec. 1999.

[14] R. Senani and S. S. Gupta, "Universal voltage-mode/current-mode biquad filter realised with current feedback op-amps," Frequenz, vol. 51, no. 7-8, pp. 203-208, July-Aug. 1997.

[15] S. S. Gupta and R. Senani, "Realisation of current-mode SRCOs using all grounded passive elements," Frequenz, vol. 57, no. 1-2, pp. 26-37, Jan. -Feb. 2003.

\section{Introduction}

Recently, in [1], the present authors proposed two novel configurations of second-order canonic $^{1}$ single-resistance-controlled oscillators (SRCO) which employ four and three unity-gain cells, respectively and offer a number of significant advantages over the previously published unity-gain follower-based oscillators of [2-10]. Note that the current interest in unity-gain-cell-based structures [11-14] as alternatives to those realized from other more complex building blocks, stems from their wider bandwidth, lower power consumption and simpler architectures.

The object of this communication is to introduce, as a sequel to [1], a new family of follower-based canonic second-order SRCOs which retain most of the advantageous features of their predecessors of [1] but, by contrast, employ a reduced number of (only two) unity-gain cells. The workability of the new SRCOs has been confirmed by PSPICE simulations using CMOSbased followers.

\section{Proposed circuits}

The proposed new SRCOs are shown in Fig. 1. The symbolic notations of VF and CFs are marked in Fig. 1 itself where the VF is characterised by the equations $i_{y}=0, v_{w}=v_{y}$ with $i_{w}$ being arbitrary and the CFs are characterised by $v_{x}=0 ; i_{z}=i_{x}$ for $\mathrm{CF}^{-}$, and $i_{z}^{+}=i_{z}^{-}=i_{x}$ for dual-output current follower (DOCF), with $v_{z}$ being arbitrary in both the cases.

\footnotetext{
${ }^{1}$ Canonic SRCOs are those which employ only three resistors and only two capacitors (which are necessary and sufficient) to achieve non-interacting controls of the frequency of oscillation as well as condition of oscillation.
} 

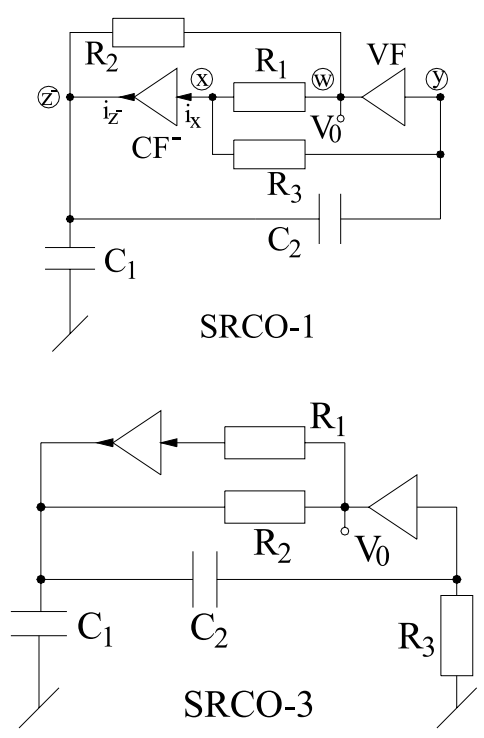
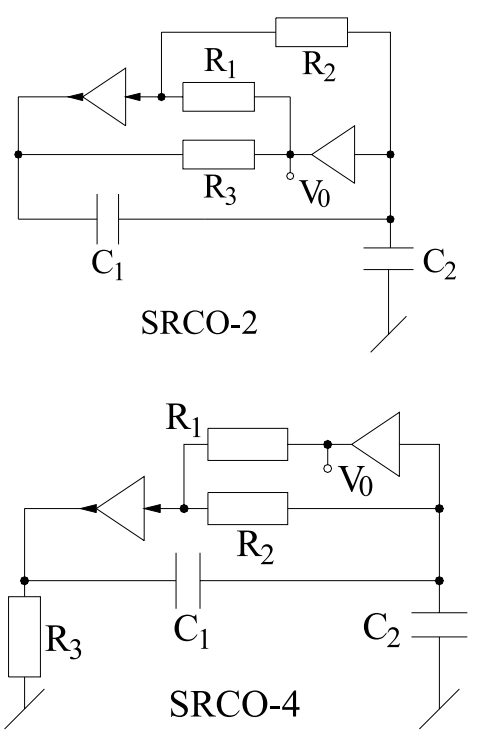

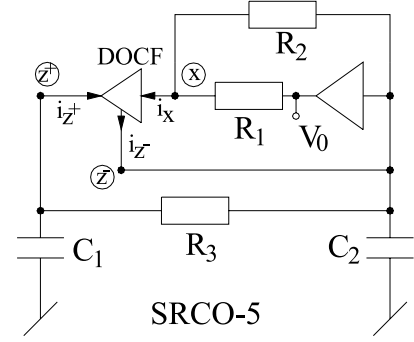

Fig. 1. Proposed new SRCOs

A straight forward analysis of the circuits gives the condition of oscillation $(\mathrm{CO})$ as:

$$
\begin{aligned}
& R_{1}=\frac{C_{2}}{C_{1}} R_{3} \text { for } S R C O-1, \quad R_{1}=\frac{C_{1}}{C_{2}} R_{3} \text { for } S R C O-2, \\
& R_{1}=\frac{C_{2} R_{3}}{C_{1}+C_{2}} \text { for } S R C O-3 \text { and } R_{1}=\frac{C_{1} R_{3}}{C_{1}+C_{2}} \text { for } S R C O-4 \text { and } 5
\end{aligned}
$$

Whereas the frequency of oscillation (FO) is given by:

$$
f_{0}=\frac{1}{2 \pi \sqrt{C_{1} C_{2} R_{2} R_{3}}} \text { for all the circuits }
$$

It is seen that $\mathrm{CO}$ and $\mathrm{FO}$ can be independently controlled through $R_{1}$ and $R_{2}$, respectively. Note that, in SRCO-5, we have used a dual-output CF instead of simple CF which has facilitated the employment of both groundedcapacitors which is attractive from integrated circuit implementation viewpoint (see [15] and references cited therein). Note that all the circuits provide an explicit voltage-mode output from a low-impedance terminal. An explicit current-mode output can also be obtained in all the cases by augmenting the $\mathrm{CF}^{-}$and DOCF with an additional current output terminal (as in SRCO-2 of Fig. 1 (c) of [1]). 


\section{Nonideal analysis and sensitivity properties}

Considering the nonideal gain of the $\mathrm{VF}$ as $\alpha$ and that of $\mathrm{CF}$ as $\beta$, the nonideal expressions of $\mathrm{CO}$ and $\mathrm{FO}$ of the SRCO-1-5 are determined and are tabulated in Table I.

Table I. Effect of nonideal parameters of the followers

\begin{tabular}{|c|c|c|}
\hline \multirow{2}{*}{ Circuit } & \multicolumn{2}{|c|}{ Nonideal expressions for CO and FO } \\
\cline { 2 - 3 } & $R_{1}=\frac{C_{2}}{C_{1}} R_{3}\left[\frac{\mathrm{CO}}{1+\frac{C_{2}}{C_{1}}(1-\beta)+\frac{C_{2}}{C_{1}}(1-\alpha) \frac{R_{3}}{R_{2}}}\right]$ & $\sqrt{\frac{2-\alpha}{C_{1} C_{2} R_{2} R_{3}}}$ \\
\hline SRCO-1 & $R_{1}=\frac{C_{1}}{C_{2}} R_{3}\left[\frac{\alpha \beta}{1+\frac{C_{1}}{C_{2}}(1-\alpha)+\frac{C_{1}}{C_{2}}(1-\beta) \frac{R_{3}}{R_{2}}}\right]$ & $\sqrt{\frac{1}{C_{1} C_{2} R_{2} R_{3}}}$ \\
\hline SRCO-2 & $R_{1}=\frac{C_{2}}{C_{1}} R_{3}\left[\frac{\alpha \beta}{1+\frac{C_{2}}{C_{1}}+\frac{C_{2}}{C_{1}}(1-\alpha) \frac{R_{3}}{R_{2}}}\right]$ & $\sqrt{\frac{1}{C_{1} C_{2} R_{2} R_{3}}}$ \\
\hline SRCO-4 and 5 & $R_{1}=\frac{C_{1}}{C_{2}} R_{3}\left[\frac{\alpha \beta}{1+\frac{C_{1}}{C_{2}}+\frac{C_{1}}{C_{2}}(1-\beta) \frac{R_{3}}{R_{2}}}\right]$ & $\sqrt{\frac{1}{C_{1} C_{2} R_{2} R_{3}}}$ \\
\hline
\end{tabular}

Thus, it is seen that after consideration of the nonideal gains of the followers, the frequency controlling resistor $\left(R_{2}\right)$ also appears in the $\mathrm{CO}$ of the proposed oscillator circuits. However, it is clear from Table I that the term containing $R_{2}$ in the nonideal expressions of the $\mathrm{CO}$ is very small due to the factor $(1-\alpha)$ or $(1-\beta)$. Additionally, this can be made even smaller if we choose $R_{2}$ to be much larger than $R_{3}$. Furthermore, note that in SRCO-1, as $S_{\alpha}^{\omega_{0}}=-\frac{\alpha}{4-2 \alpha}$ which approaches $1 / 2$ as $\alpha \rightarrow 1$, the presence of $\alpha$ does not alter the value of $\omega_{0}$ significantly. On the other hand, SRCOs-2-5 are completely unaffected by the nonideal gains $\alpha$ and $\beta$ of the followers.

\section{Frequency stability properties}

The frequency stability factor $S_{F}=\left.\frac{d \phi(u)}{d u}\right|_{u=1}$ (where $u=\frac{\omega}{\omega_{0}}$ is the normalized frequency and $\phi(u)$ represents the phase function of the open loop transfer function) with $C_{1}=C_{2}=C$ and $R_{1}=R_{3}=R$ and $R_{2}=R / n$, is found to be $S_{F}=\frac{\sqrt{n}}{n+1}$ for SRCO-1, $S_{F}=\frac{2 \sqrt{n}}{n+1}$ for SRCO-3 and $S_{F}=\sqrt{n}$ for SRCO-2, 4 and 5 . Thus, it is seen that by keeping ' $n$ ' large, SRCO-2, 4 and 5 would have high values of $S_{F}$ and therefore, enjoy very good frequency stability properties.

\section{Simulation results}

For the simulation of the proposed SRCOs in PSPICE, we have used the CMOS VF and CFs as in [1]. The SRCOs were designed to have $f_{0}=1 \mathrm{MHz}$ by choosing component values as $R_{2}=R_{3}=4 \mathrm{k} \Omega, C_{1}=C_{2}=39.8 \mathrm{pF}$ and $R_{1}$ was adjusted to meet the $\mathrm{CO}$. We present here some sample results for the 
proposed circuits. PSPICE generated transient response of SRCO-1 is shown in Fig. 2 (a) whereas the variation of $f_{0}$ (from $1.6 \mathrm{MHz}$ to $0.21 \mathrm{MHz}$ ) with $R_{2}$ varied from $1 \mathrm{k} \Omega-100 \mathrm{k} \Omega$ for SRCO- 1 , using $R_{3}=4 \mathrm{k} \Omega, C_{1}=C_{2}=39.8 \mathrm{pF}$, is shown in Fig. 2 (b). Simulation results of Fig. 2, thus, confirm the workability of the proposed configurations.

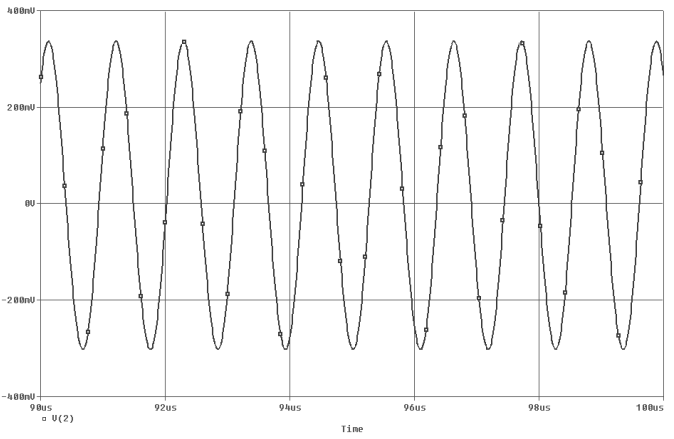

(a)

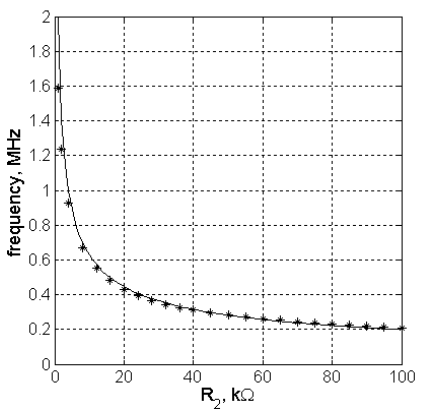

(b)

Fig. 2. (a) Transient response of SRCO-1 $\left(f_{0}=0.93 \mathrm{MHz}\right.$; $550 \mathrm{mV}$ p-p)

(b) Variation of $f_{0}$ with $R_{2}$ for SRCO-1 $(1.6 \mathrm{MHz}-$

$0.21 \mathrm{MHz}$ for $R_{2}=1 \mathrm{k} \Omega-100 \mathrm{k} \Omega$ )

\section{Concluding remarks}

In a recent publication [1] the authors have proposed two new SRCO configurations which were realised from only four/three followers and provided a number of advantages as compared to the previously known follower-based oscillators of [2-10]. In this communication, we have introduced a number of new follower-based oscillators which offer the following advantages:

(i) are second-order single-resistance-controlled oscillators

(ii) provide explicit VM output and can provide explicit $\mathrm{CM}$ output with an additional current output terminal in the used $\mathrm{CF}$

(iii) are canonic in number of passive elements, and

(iv) enjoy very low sensitivity property. 
Furthermore, three of the new circuits (namely SRCO-2, 4 and 5) have large frequency stability factor like their predecessors of [1] and one of these circuits (namely SRCO-5) has the additional feature of employing both groundedcapacitors as preferred from the viewpoint of IC implementation (see [15] and references cited therein).

Thus, the new circuits retain most of the advantages of their predecessors of [1] while requiring a reduced ${ }^{2}$ number of (only two) followers.

\footnotetext{
${ }^{2}$ Discovering any 'second-order canonic SRCO employing no more than a single CF or a single $\mathrm{VF}^{\prime}$ ' appears to be a very challenging problem!
} 